\title{
La utilización del espacio en la ocupación de la cueva de Praileaitz I (Deba, Gipuzkoa, Euskal Herria)
}

\author{
Espazioaren erabilera Praileaitz I haitzuloaren (Deba, Gipuzkoa, Euskal Herria) \\ bizitze garaian
}

\author{
The usage of the space in the occupation of the Praileaitz I cave \\ (Deba, Gipuzkoa, Euskal Herria)
}

PALABRAS-CLAVE: Euskal Herria, Ocupación del espacio, Actividad ritual, Pintura rupestre.

GAKO-HITZAK: Euskal Herria, Espazioaren okupazioa, Jarduera errituala, Labarretako margoa.

KEYWORDS: Basque Country, Space occupation, Ritual activity, Cave painting.

Xabier PEÑALVER(1), Sonia SAN JOSE(2), Jose Antonio MUJIKA-ALUSTIZA ${ }^{(3)}$

\section{RESUMEN}

La ocupación espacial de la cueva de Praileaitz I (Deba) presenta diferencias y semejanzas en función de los distintos momentos en que ha sido habitada por el ser humano. Aquí se hace un recorrido del uso dado a cada uno de los espacios en los que se divide la cavidad a partir del período Gravetiense hasta el abandono del lugar en el Epipaleolítico, destacando por su importancia la fase correspondiente al Magdaleniense Inferior.

\section{LABURPENA}

Praileaitz I kobako (Deba) guneen okupazioak ezberdintasunak eta antzekotasunak ditu gizakiak bertan bizi izan diren garaien arabera. Hemen, haitzuloa banatzen den gune bakoitzaren erabileraren ibilbidearen azalpena egiten da, hasi Gravette aldian eta Epipaleolito Aroan utzi zuten arte, garai horien artean Behe Madeleine aldia nabarmentzen delarik.

\section{ABSTRACT}

The spatial occupation of the Praileaitz I cave (Deba) shows differences and similarities depending on the moments in which it was inhabited by human beings. In this paper, the usages that the different spaces of the cavity had from the Gravetian to its abandonment in the Epipaleolithic are reviewed. The importance of the Lower Magdalenian period is highlighted.

La excavación del yacimiento de Praileaitz I a lo largo de una década se ha extendido a zonas muy amplias del mismo, lo que ha permitido profundizar en las características de las efímeras ocupaciones que se han sucedido a lo largo del Paleolítico Superior (Solutrense, distintas fases del Magdaleniense) y Epipaleolítico (PEÑALVER, 2014b, PEÑALVER et al., 2017). Sin embargo, hay que señalar que no se ha alcanzado la roca madre en ninguna de las áreas de la cavidad, a excepción de en la plataforma exterior existente a un lado de la entrada.

Las intervenciones reiniciadas en 2012, y que no son objeto de estudio en esta memoria, se centran en la zona noroeste del vestíbulo (cuadros 6F y 8F), en la segunda sala interior (cuadros 12J', 14J' y 16J'), y sobre todo en la prolongación de la galería noroeste hacia el oeste (PEÑALVER, 2013 y 2014a). El objetivo principal de estos trabajos es identificar aquellas ocupaciones anteriores al Solutrense, y más concretamente el origen de algunos restos de aspecto musteriense cuya presencia se ha constatado en el vestíbulo, en el contacto con la galería noroeste.

La potencia de los diferentes niveles varía según la zona. El mayor espesor se detectó en el vestíbulo (Fig. 1), donde los sedimentos procedentes de la "chimenea" allí existente, y aquellos otros acarreados por escorrentía ladera abajo se acumulaban principalmente bajo el dintel. Prueba de ello es que el nivel de colgantes atribuible al Magdaleniense Inferior se detecta en el vestíbulo a -129 $\mathrm{cm}$ bajo la superficie (cuadro 10G) mientras que en el tránsito de aquí a la primera sala se sitúa a -51 cm (cua-

\footnotetext{
(1) Departamento de Arqueología Prehistórica. Sociedad de Ciencias Aranzadi. Zorroagaina, z.g. 20014 Donostia. praileaitz@gmx.com (2) Departamento de Arqueología Prehistórica. Sociedad de Ciencias Aranzadi

(3) Facultad de Letras (UPV-EHU)
} 


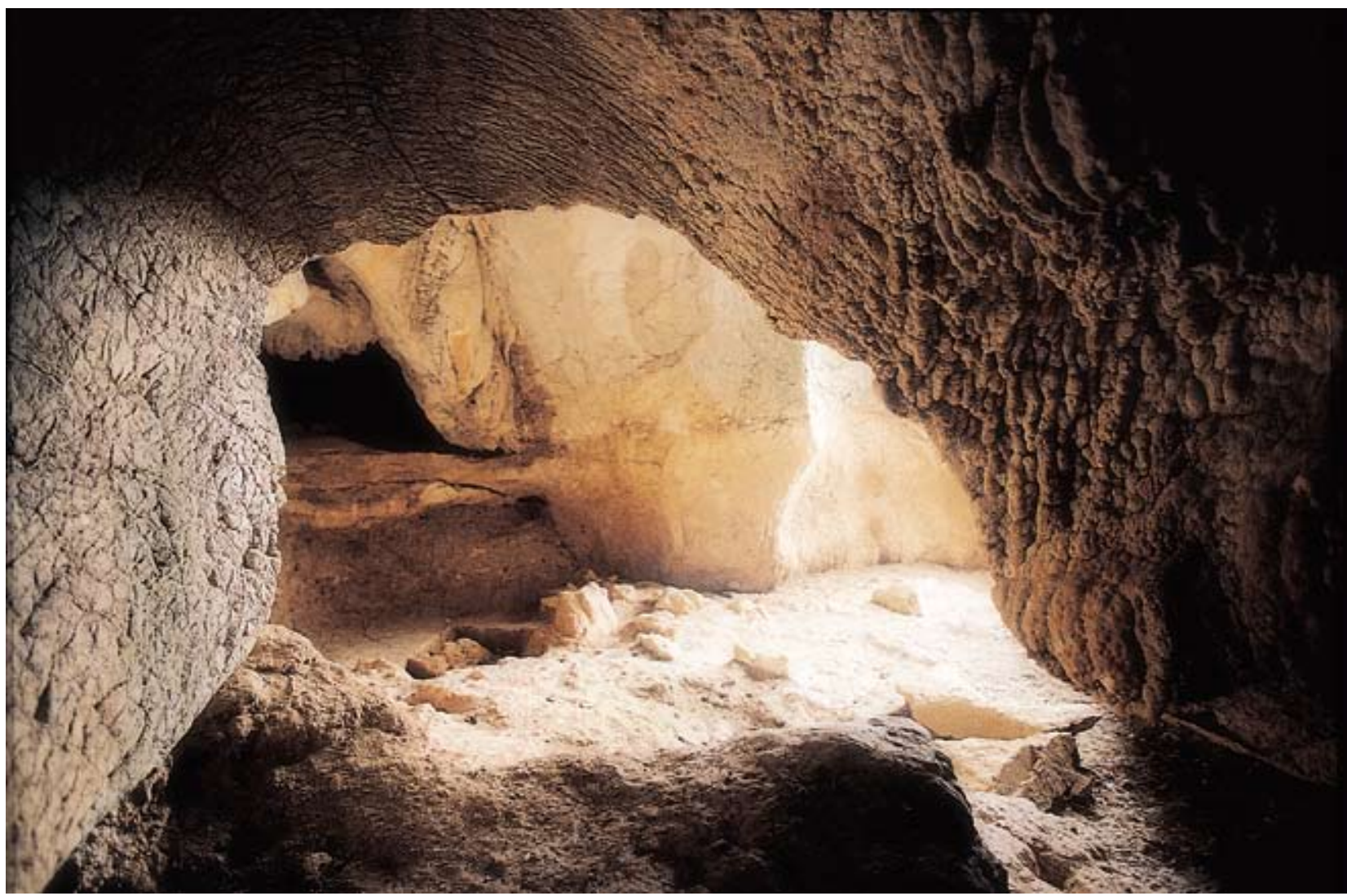

Fig. 1. Vestíbulo iluminado por la cercana boca de la cueva (X. Otero). / Vestibule iluminated by the near entrance of the cave (X. Otero).

dro 12B) y a tan solo $-20 \mathrm{~cm}$ bajo la capa de concreción que cubría esta sala.

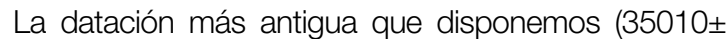
280 BP) es la obtenida a partir de una ulna de Pantera pardus, hallada en el rellano exterior existente bajo un pequeño abrigo ubicado a un lado de la entrada a la cueva en la que se han excavado $16 \mathrm{~m}^{2}$. Los restos faunísticos aquí eran frecuentes, pero no así los de carácter industrial que eran escasos y poco característicos, siendo reseñable únicamente la detección de alguna fugaz ocupación humana durante el Aziliense o el Magdaleniense Final.

Es el vestíbulo y su entorno -zona de tránsito a la primera sala interior- el sector de Praileaitz I que más vestigios estratificados ha aportado, aunque todos ellos corresponden a fugaces y recurrentes ocupaciones. Cronológicamente, las dos dataciones más antiguas $(25320 \pm 140$ y $22900 \pm 110 \mathrm{BP})$ nos retrotraen al Gravetiense, aunque por ahora no se han identificado restos industriales característicos de esta época, por lo que tampoco se puede descartar que se relacionen con restos de animales ajenos a la ocupación humana. También hay que indicar que no se ha profundizado en extensión, hasta la fecha, en cotas inferiores del vestíbulo (por debajo de los primeros indicios solutrenses), y los restos que indican mayor antigüedad se han localizado en el contacto entre éste y la galería noroeste. Ésta última galería estaba casi colmatada en su totalidad y sellada por una potente capa estalagmítica, constatándose ocasionalmente objetos de sílex, quizás descontextualizados o en posición secundaria, y cuyo origen habría que buscar en el otro extremo de la galería. Por otra parte, no es descartable que en el vestíbulo hayan desaparecido parte de estos niveles más antiguos a causa de un vaciado posterior.

El Solutrense es uno de los periodos culturales mejor representados (Figs. 2 y 3), a pesar de no haberse excavado en su totalidad en amplias zonas de la cueva, gracias a la presencia de un número significativo de objetos muy característicos, de retoques planos, en las distintas zonas de la cavidad (vestíbulo, primera sala interior y extremo norte de la segunda sala interior). Esta cronología se ve sustentada también por varias dataciones $(19330 \pm 150,17760 \pm 70$ y $17530 \pm 70 \mathrm{BP})$. Hay que advertir que estos restos estaban en contacto, casi entremezclándose, con los de las ocupaciones del Magdaleniense Inferior que se le superpone.

La ocupación de la cavidad durante el Magdaleniense Inferior está datada en torno al $15500 \mathrm{BP}$, y es sin duda la más importante de todas las detectadas hasta hoy en Praileaitz I, y no sólo por la relevancia de los materiales hallados, sino por la ordenación del espacio en 


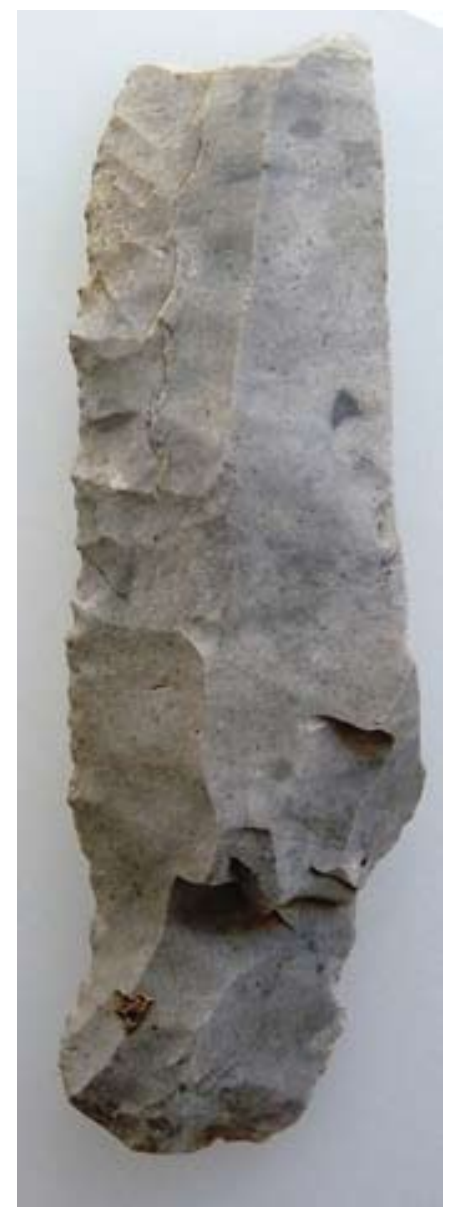

ese momento, y su estructuración en torno a un hogar, un posible asiento y sobre un suelo empedrado intencionadamente (Figs. 4 y 5). Los 29 colgantes recogidos en las distintas zonas de la cueva son un claro testimonio del área frecuentada, aunque la estancia, al igual que la de otros momentos cronológicos, parece que fue temporal y no muy duradera, si nos basamos en el número y características de los restos arqueológicos recuperados. El hogar parece responder a una fogata, o a una serie de ellas prendidas en un breve periodo en el mismo punto. No se observaron hogares yuxtapuestos, ni superpuestos a distintas cotas, como se ha descrito en otros asentamientos estacionales y como sucede en los niveles VI y VII de Ekain, donde se determinaron distintas situaciones: así, se detectaron hasta cuatro hogares, uno de ellos tras preparar una pequeña hondonada y junto al mismo había "un gran bloque apto para sentarse" además de casi todos los útiles, así como numerosas lascas y restos faunísticos. En el más antiguo, que estaba cerca de la pared interior, existía una acumulación de clastos y allí se concentraban también la mayoría de las piezas (ALTUNA, MERINO, 1984: 33-34).

Las áreas más intensamente frecuentadas a lo largo del Magdaleniense Inferior fueron el vestíbulo -la zona más luminosa-, la primera sala interior -de un ambiente en penumbra- y el "túnel" de tránsito entre estos dos espacios, de aproximadamente $1 \mathrm{~m}$ de altura y 1,50 $\mathrm{m}$ de anchura. La totalidad de esta superficie comprende 89 $\mathrm{m}^{2}$ y presenta características diferentes según el sector.

El vestíbulo, con una superficie de $34 \mathrm{~m}^{2}$, muestra un suelo relativamente horizontal -la diferencia máxima de las cotas de los ejes longitudinal y transversal se sitúa en unos $20 \mathrm{~cm}$ - formado por pequeños clastos de calizas que parecen circunscribirse a él, ya que apenas se adentran en la galería noroeste y en la zona de tránsito a la primera sala interior (PEÑALVER, MUJIKA, 2003 y 2005b). Los cantos muestran unas dimensiones muy homogéneas (de unos $10 \mathrm{~cm}$ ), llegando a constituir una capa irregular de unos $3 \mathrm{~cm}$ de espesor (subnivel IV.II). Probablemente, la colocación de carácter antrópico de estos clastos tuvo la intencionalidad de preparar un piso bien drenado, y pudieron haber sido recogidos en el entorno de la propia cavidad, donde no faltan zonas abruptas a cuyos pies se pudieron formar acumulaciones de clastos.

La existencia de suelos preparados en asentamientos magdalenienses está documentada en varios yacimientos. En la cueva de Isturitz, en la base del nivel magdaleniense de la Sala San Martín, se describe una capa casi continua de piedras planas de dimensiones variables (25-30 cm), cuyo carácter antrópico no se señala, pero que quizás no habría que desechar (SAINT-PÉRIER, 1930). También al norte de los Pirineos podemos citar en el Magdaleniense Superior-Final de Bourrouilla (Arancou) la existencia de un hogar y una estructura subcircular conformada por losas calizas, al parecer relacionada con actividades de fabricación y arreglos del instrumental de caza (DACHARY et al., 2008).

En los yacimientos de la falaise de Pastou también se señalan otras estructuras. En concreto en Duruthy, 


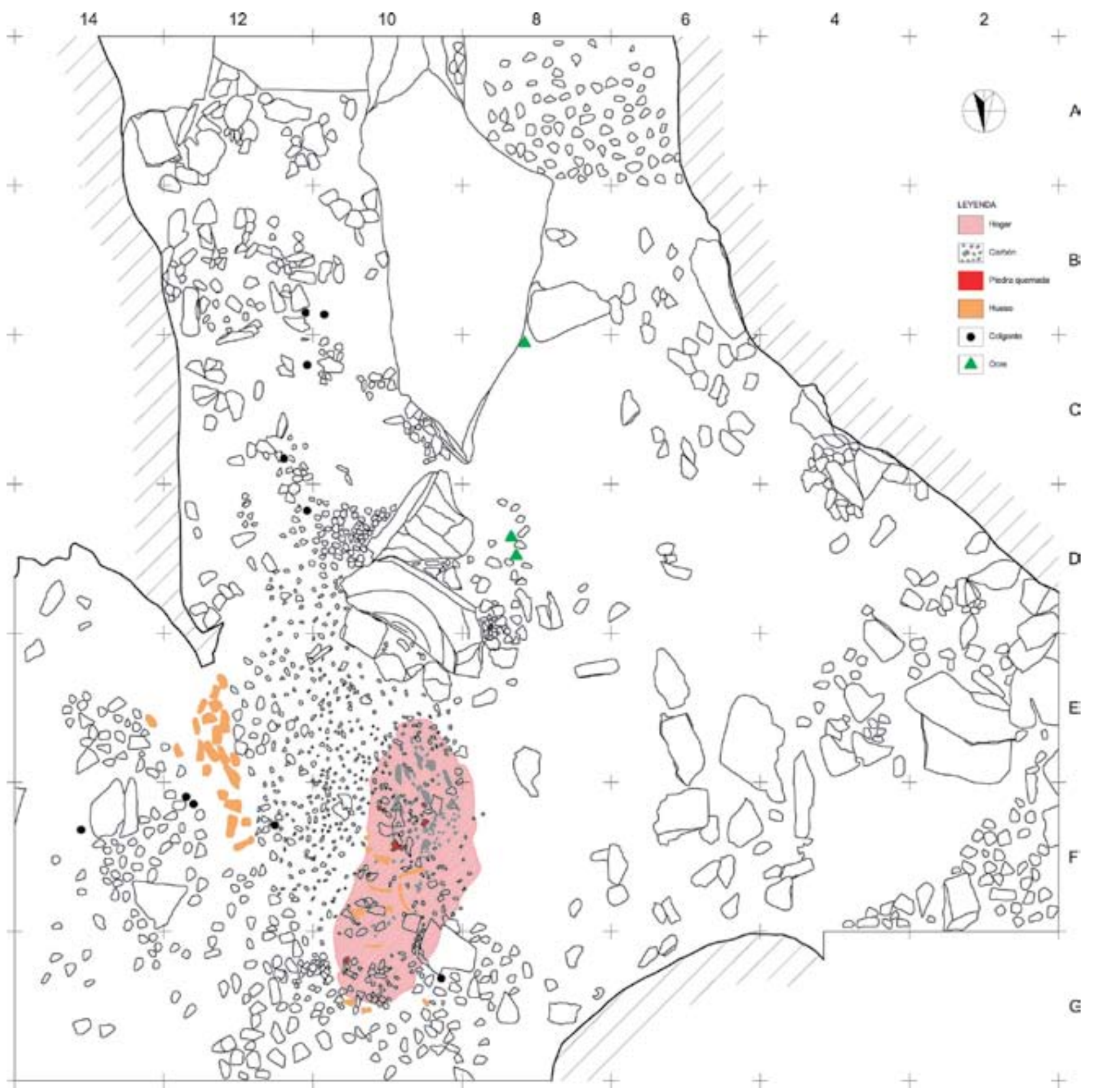

Fig. 4. Nivel del Magdaleniense Inferior en la zona del vestíbulo y detalle del hogar y el asiento del mismo nivel (Dibujo X. Peñalver/Digitalización J. Calvo). / Level of the Lower Magdalenian in the vestibule area and detail of the hearth and the seat at the same level (Drawing X. Peñalver/Digitalisation J. Calvo).

Arambourou (1978, 147 ss) describe en el Magdaleniense VI el transporte de cantos rodados, entre los que se intercalan otros restos desprendidos de las paredes y trozos de plaquetas de arenisca, para sanear y crear un encachado, además de algún murete con bloques dipuestos de canto, y sobre todo trabajos de acondicionamiento del pronunciado talud existente bajo la falaise, creando al menos seis aterrazamientos. En estos encachados se han observado, además, agujeros de postes a fin de construir alguna estructura parcialmente aérea (tienda, paravientos, etc.) (p. 27 ss.).
En los niveles del Magdaleniense IV (p. 47 ss, 148), en las proximidades de un hogar, se apreciaron abundantes plaquetas de arenisca, algunas enrojecidas por el fuego, que pavimentaban el suelo, aunque el sedimento arcilloso y la caída de bloques han modificado su disposición original. Hay también tenues indicios de una distribución espacial de actividades, así la parte baja del talud, al parecer, servía de vertedero. Finalmente, en dos de las ocupaciones del Magdaleniense III (pág. 60 ss), detectadas en siete niveles, se apreciaron trabajos de acondicionamiento del hábitat (cantos delimitando probablemente hogares, etc.). 
12
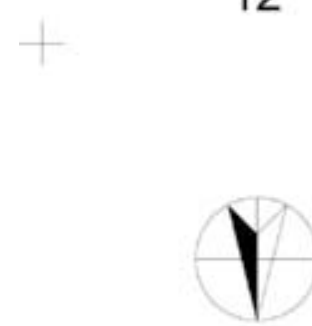

10

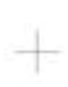

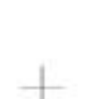

8

\section{LEYENDA}

$\because \because$ Carbón

Piedra quemada

Hueso

Azagaya

\section{$+$}
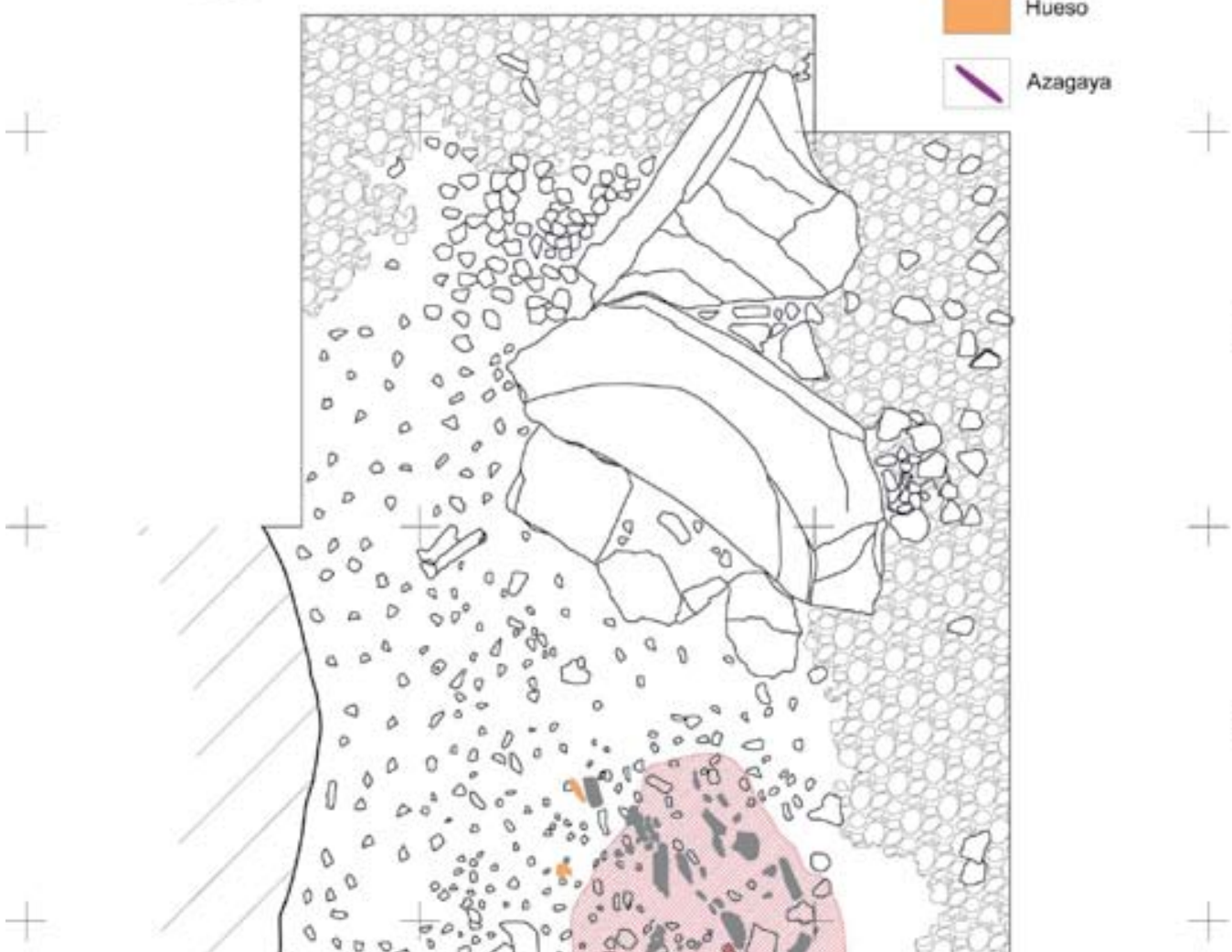

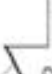

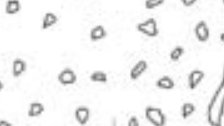

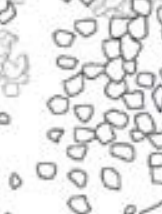

0.000

$\therefore 000$

0,0 ,

0.0 .8000

$0=0.0 \%$

$\circ \quad 000000$

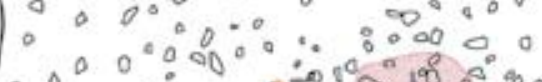

$0_{0}^{\circ} 0^{\circ} 18 \mathrm{a}^{\circ}, 0_{0}$

000

400

$\therefore 8: \circ 8$

$20^{\circ}: 0,005$

$\int_{0} \int_{0}^{0}$

00

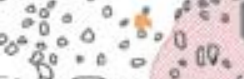

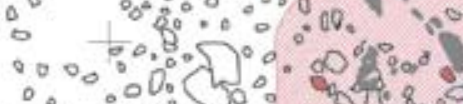
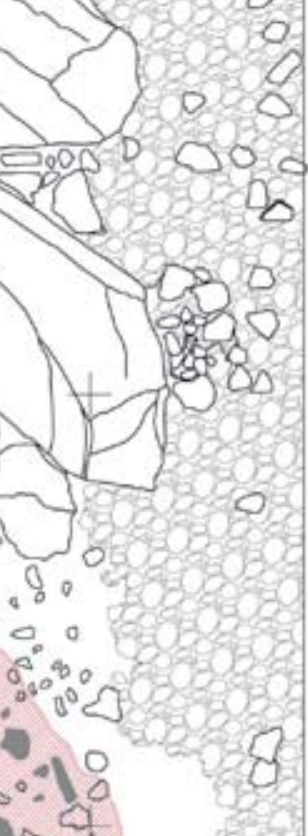

- $0.0 ; 000$

$\therefore \circ 00: 000$ ?

$00^{\circ}$
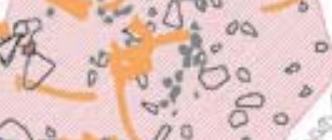

0.000

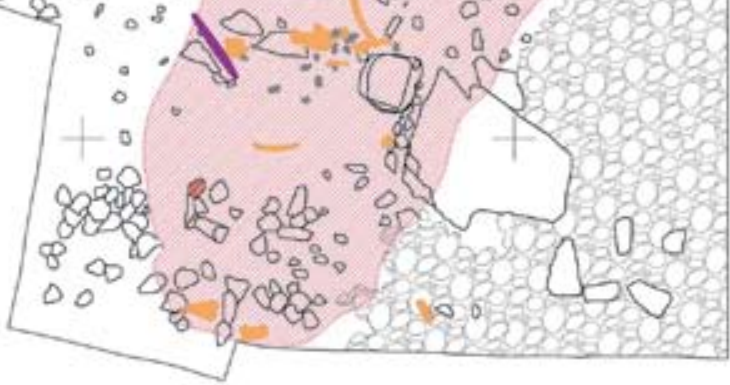

E

Fig. 5. Detalle del hogar y del asiento. / Detail of the hearth and the seat. 
Fig. 6-7. Dos aspectos de la piedra utilizada como asiento ante el hogar en la entrada del vestíbulo, durante el Magdaleniense Inferior (E. Koch). / Two views of the stone used as a seat in front of the hearth at the entrance of the vestibule, during the Lower Magdalenian (E. Koch).

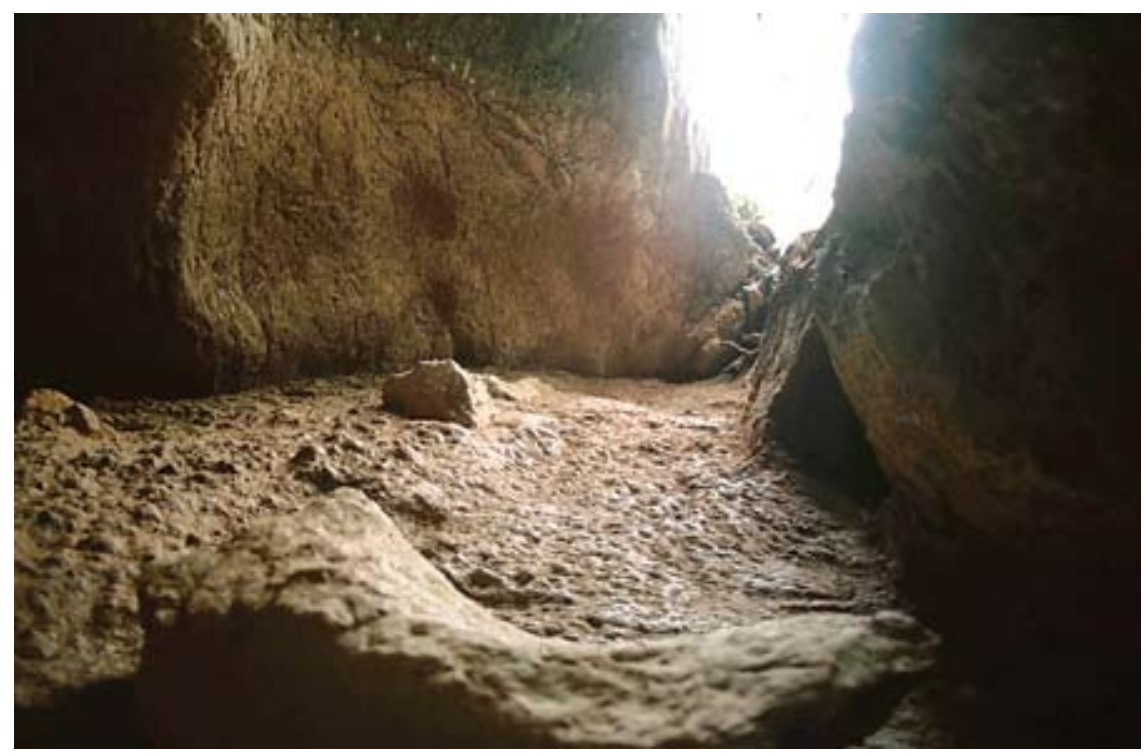

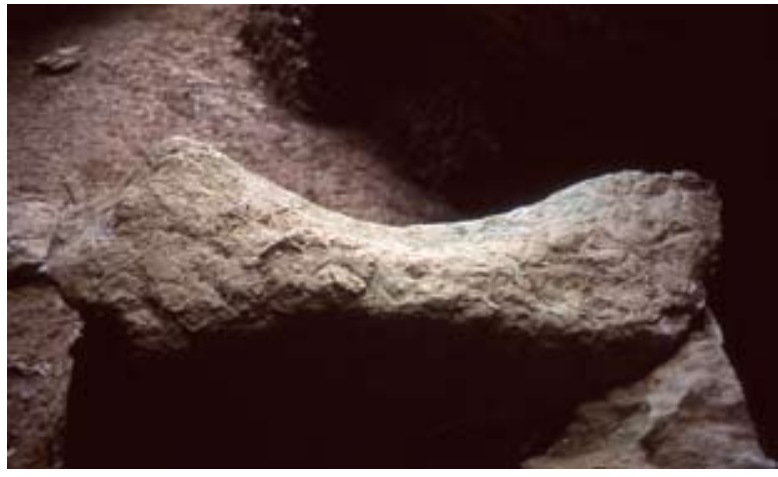

En las proximidades de Duruthy, se localizan los abrigos de Dufaure y Grand Pastou donde se han identificado sucesivos empedrados (hasta ocho en el nivel 4) constituidos por plaquetas de arenisca durante el Magdaleniense, y por cantos rodados posteriormente (DACHARY, 2006). En determinadas zonas se observaban finas capas de limos que separarían periodos de desocupación, así como pavimentos intensamente quemados a veces por haber crecido vegetación en dichos periodos, o fuegos para el tratamiento de la caza, etc. (STRAUSS, 1998).

En el conjunto de la Garma se han señalado estructuras en la Galería Inferior y en La Garma A atribuibles al Magdaleniense Medio. En esta última se aprecia una estructura, una alineación ligeramente curvada, oblicua respecto del eje de la galería, que delimita un espacio oval, de 2,4 X 1,7 m aproximadamente, contra la pared. Dentro de ella existe una menor densidad de restos arqueológicos, tanto líticos como óseos, que en la zona aledaña y, también, más fracturados. Entre la cara interior del paramento y la pared meridional de la sala aparece una acumulación de areniscas teñidas de ocre. También se observa en algunos sectores, en un nivel de limos amarillentos profusión de inclusiones de caliza y estalagmita de fracción canto, que pueden atribuirse a la intervención humana (ARIAS et al., 2005).

En la Galería Inferior (ONTAÑON, 2003), dividida en 4 zonas, se detectaron al menos 6 estructuras artificiales y abundantes restos asociados a ellas: miles de huesos en superficie (a veces formando acumulaciones arrinconadas junto a las paredes -posibles zonas marginales-), abundante industria lítica y ósea, manifestaciones de arte parietal, restos de iluminación, etc. Algunas de las estructuras están construidas con bloques junto a las paredes de la cavidad y se señalan diferencias entre las características del área interior (más compacto y con menos restos) y sus alrededores.

Más al occidente, en el nivel II de Las Caldas, S. Corchón $(1995,6)$ señala que "la concentración de cantos calizos de estas características pudiera revestir carácter antrópico, dada su regular disposición, la selección en el tamaño de los cantos y la abundancia de otros de cuarcita, en relación con la existencia de un hogar conservado en el cuadro $\mathrm{H}-4$ y construido con ellos".

Volviendo a la cueva de Praileaitz I, en el vestíbulo, el espacio más amplio y luminoso de la cavidad, asimismo en el nivel correspondiente al Magdaleniense Inferior, se sitúan tres bloques calizos de notables dimensiones, relativamente alineados entre sí, que delimitan dos sectores: un cubículo, situado al este, y el resto del vestíbulo. El mayor de los bloques, que dificulta el paso desde el vestíbulo a la primera sala interior, pudo haberse desprendido del techo.

Los otros dos bloques que ocupan junto al hogar un espacio casi central en el vestíbulo, son de dimensiones más discretas, y el menor de ellos sirve de calce al otro que tiene forma aquillada, de 1,10 x 0,43 m y 0,20 m de espesor. Este pudo haber sido un asiento (Figs. 6 y 7), ya que se sitúa frente a un hogar en cubeta, claramente delimitado, de unos $2 \mathrm{~m}$ de largo y 0,80 m de ancho, y con abundantes restos de carbón, huesos de fauna y piedras 
afectadas por el calor; ante el posible asiento, una serie de piedras planas, situadas de forma alineada, podrían haber servido para colocar los pies. En las proximidades de este hogar se halló una azagaya de base hendida, de sección circular, con paralelos en Santimamiñe y Balmori (PEÑALVER, MUJIKA, 2005a; VEGA DEL SELLA, 1930: Fig. 44). A espaldas del bloque, y arrinconada junto a la pared, se localizó una importante concentración de restos de fauna en conexión anatómica.

La presencia de bloques susceptibles de ser utilizados a modo de asiento se ha propuesto también en Erralla, donde alrededor de un gran bloque prismático se detectó una importante concentración de restos industriales y óseos (ALTUNA, BALDEÓN, MARIEZKURRENA, 1985).

El cubículo de Praileaitz I alcanza una superficie de $4 \mathrm{~m}^{2}$ y su altura respecto del techo es de apenas $80 \mathrm{~cm}$, lo que limita el normal tránsito por dicho espacio durante el desarrollo de las actividades cotidianas. De hecho las industrias sobre sílex son muy escasas aquí, tratándose principalmente de productos de talla. Ya en contacto con la zona de tránsito a la primera sala interior se recogió un canto rodado de forma ovalada, aparentemente sin manipular, en una roca similar a la de los colgantes de este nivel. Sin embargo, en este pequeño espacio se han hallado, muy próximos entre sí, varios objetos ya acabados, concretamente cinco colgantes. Tres son incisivos de cabra con doble perforación en su raíz y decorados con finos cortes transversales dispuestos en su cara labial -con restos de ocre-, y dos colgantes sobre cantos rodados de lutita muy oscura -negra o grisácea-, cuyo tono oscuro y brillo se acentúan notablemente al aumentar su humedad. Uno de ellos es de sección muy aplanada y de forma romboidal, y muestra varias finas incisiones transversales en una de sus caras, evocando la silueta de muchas de las venus paleolíticas (Fig. 8 y 9); el otro, por lo contrario, un cuerpo voluminoso, periforme, decorado con incisiones transversales dispuestas en paralelo a distancias regulares, que recuerda indudablemente a un canino atrofiado de ciervo, aunque de mayores dimensiones.

Dentro del vestíbulo, además, se puede diferenciar por un lado el subsector situado al fondo y la bifurcación

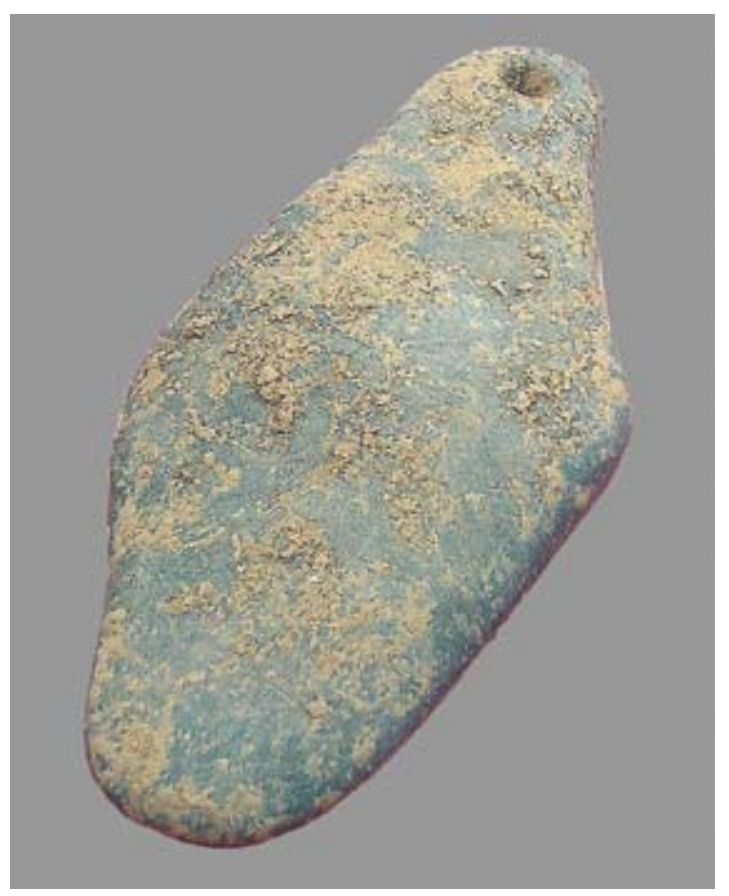

Fig. 8. Colgante $n^{\circ} 1$ en el momento del hallazgo. / Pendant no. 1 at the moment of the discovery.
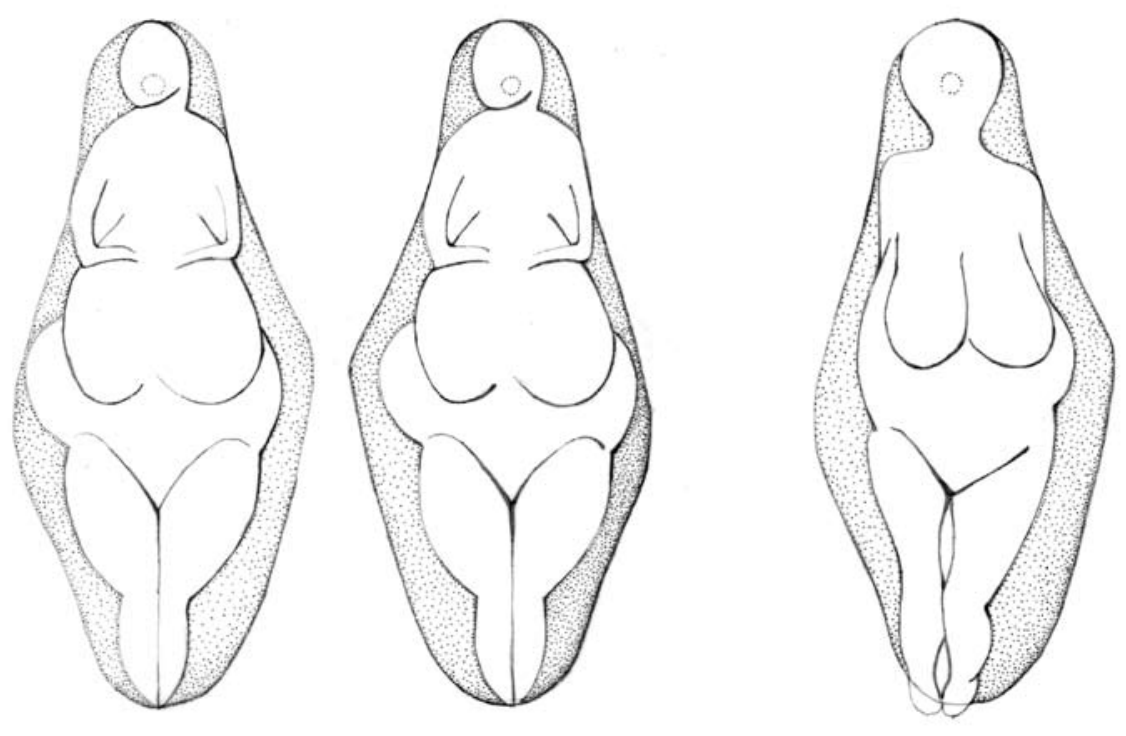

Fig. 9. Colgante $n^{\circ} 1$ de Praileaitz I con superposición, tras adaptar las escalas, de las venus de Lespugue (izquierda) y Kostienki (derecha) realizadas por $\mathrm{A}$. Leroi-Gourhan (1984: 514). / Pendant no. 1 from Praileaitz I with superposition, after adapting the scales, of the images of the venuses of Lespugue (left) and Kostienki (right) made by $\mathrm{A}$. Leroi-Gourhan (1984: 514). 


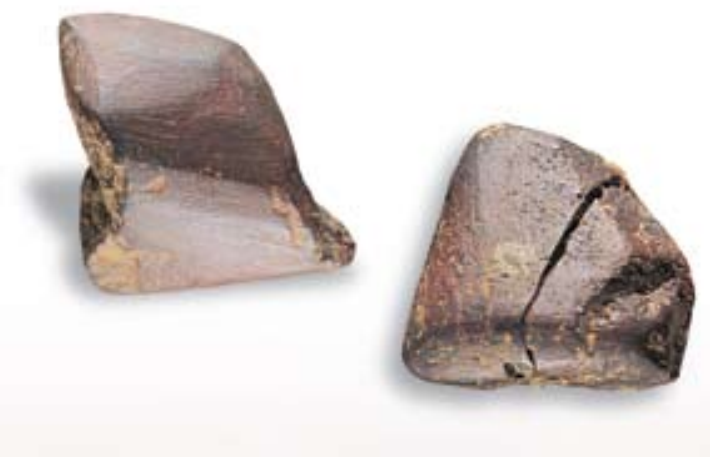

donde se inicia una galería lateral, y por otro, el espacio ubicado bajo el actual umbral de la cueva.

La zona del fondo del vestíbulo, a pesar de que aparentemente ofrece características propicias para el desarrollo de actividades cotidianas, parece sin embargo un área marginal a la luz de los escasos restos recuperados. Sin embargo, destaca la presencia de seis trozos de ocre, algunos brutos, varios de los cuales tienen huellas de utilización a modo de estrías longitudinales de abrasión (dos de estos últimos encajan entre sí) (Fig. 10); en esa misma zona se ha recuperado un fragmento medial de costilla de animal decorada con un haz de incisiones paralelas en una de sus caras dentro del cuadro 6C.

En cuanto al subsector exterior del vestíbulo situado bajo el umbral de la entrada y en el entorno del hogar (cuadros 10E y 10F), se hallaba aparentemente protegido por un gran bloque calizo desprendido de la pared este de la entrada. La excavación permitió probar que este bloque habría caído directamente sobre uno
Fig. 10. Lápices de ocre hallados en el nivel Magdaleniense Inferior del vestíbulo (X. Otero). / Ochre pencils found at the Lower Magdalenian level of the vestibule $(X$. Otero). de los colgantes y una acumulación de huesos próxima a la pared señalada anteriormente (Fig. 11), por lo que la morfología de esta entrada debió de ser distinta de la actual.

El colapso del antiguo dintel de la entrada pudo ser casual e independiente de otros fenómenos similares que se producian recurrentemente, pero también pudo haber coincidido con los episodios de caídas de bloques o desplomes de techos documentados en algunas cuevas a finales del Magdaleniense Inferior (UTRILLA, 1990: 47). Así, bajo el Magdaleniense Superior de Cueto de la Mina y en el Magdaleniense Medio, según descripción del Conde Vega de Sella, se detectaban hacia el exterior, junto a la pared del lado oeste, sedimentos que indican un episodio de caída de bloques y deslizamiento de arcillas (CHAPA, 1975). En el nivel Lgc de Antoliña, parcialmente coincidente con esta fase, existen fenómenos de solifluxión y de mayor humedad en algunos cuadros, reforzado este dato por el estudio antracológico provisional

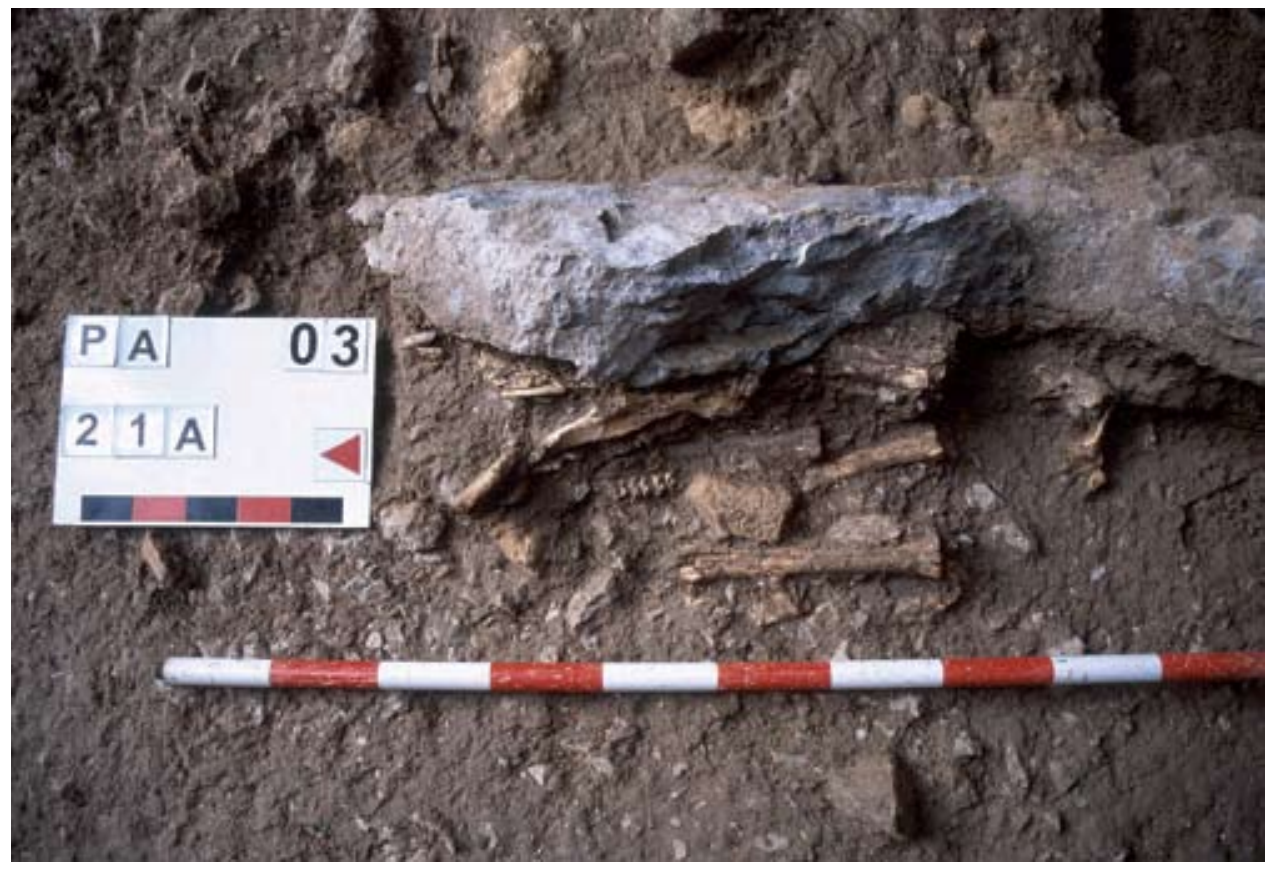

Fig. 11. Concentración de restos de fauna bajo los últimos fragmentos del gran bloque calizo desprendido de la pared de la entrada de la cueva durante el Magdaleniense Inferior. / Concentration of fauna remains under the last fragments of the great limestone block detached from the wall at the entrance of the cave during the Lower Magdalenian. 
que señala el máximo de roble en la secuencia (AGUIRRE, 2000; AGUIRRE et al., 2000: 20).

En Praileaitz I, en el subsector exterior del vestíbulo los restos de industria lítica son más abundantes que en otras zonas, destacando la presencia de 6 puntas de dorso, 2 fragmentos mediales de laminillas de dorso, 1 buril, 1 raedera y 1 escotadura, y unos pocos productos de talla. Además de estas industrias, se recogieron una azagaya con bisel liso ricamente decorada y cuatro colgantes de piedra -uno partido en dos al haber caído sobre él el gran bloque desprendido del umbral y pared de la entrada-, dos de ellos decorados con cortos trazos transversales, de características similares a los hallados en el cubículo.

La dispersión de los útiles y restos de talla indica que a pesar de su pobreza la mayor densidad se da alrededor del hogar, en la zona central del vestíbulo. Esto mismo se había señalado con anterioridad, por ejemplo, en las distintas ocupaciones de Ekain (MERINO, 1984: 156-158) y concretamente en las magdalenienses. De la dispersión de la industria lítica del nivel VII de Ekain, señalaba que ésta era tallada alrededor de los hogares, y que los buriles y los raspadores tendían a estar alejados de ellos. Lamentablemente, la escasez de objetos en el caso de Praileaitz I imposibilita detallar este aspecto.

El análisis de los restos de esta ocupación del Magdaleniense Inferior indica que hay un profundo desequilibrio entre el número de restos de talla y el de objetos retocados y abandonados -principalmente los dorsos-. Además, entre los primeros, se observa la ausencia de fases teóricas de una cadena operativa de talla (carencia de restos corticales, de lascas de reacondicionamiento de núcleos y de útiles, etc.). No hay una continuidad lógica entre los diferentes tipos de restos por lo que deducimos que los instrumentos líticos fueron aportados ya elaborados desde otro asentamiento. Lo mismo parece deducirse de los objetos óseos y de los colgantes, por la ausencia de fragmentos de lengüetas o de matrices y escasez de buriles y perforadores.

La continuidad entre el vestíbulo y la primera sala interior, separadas físicamente por un estrecho pasadizo, es evidente por la existencia de objetos y acciones compartidas. La citada sala, cuya superficie estaba cubierta por una gruesa capa estalagmítica, es de forma casi circular, de $7 \mathrm{~m}$ de diámetro y una altura máxima de aproximadamente 2,25 m, y de 1,60 m en la zona en la que se descubrió un alineamiento de 14 colgantes líticos decorados con sencillos temas de inspiración geométrica (en torno al cuadro 18E'), similares a los del vestíbulo. Estas piezas probablemente formaban parte de un mismo collar, que guardaría la posición en la que fueron abandonadas. Además, se hallaron dispersos en el resto de la sala otros 6 colgantes: 2 en la zona central (cuadro 14F') y uno algo más al sur (cuadro 16G'), así como otros 3 rotos a la altura de la perforación (cuadros 8D', 8F' y $\left.10 F^{\prime}\right)$, además de un fragmento de uno de los colgantes descubierto en la entrada del vestíbulo (cuadro 8G').

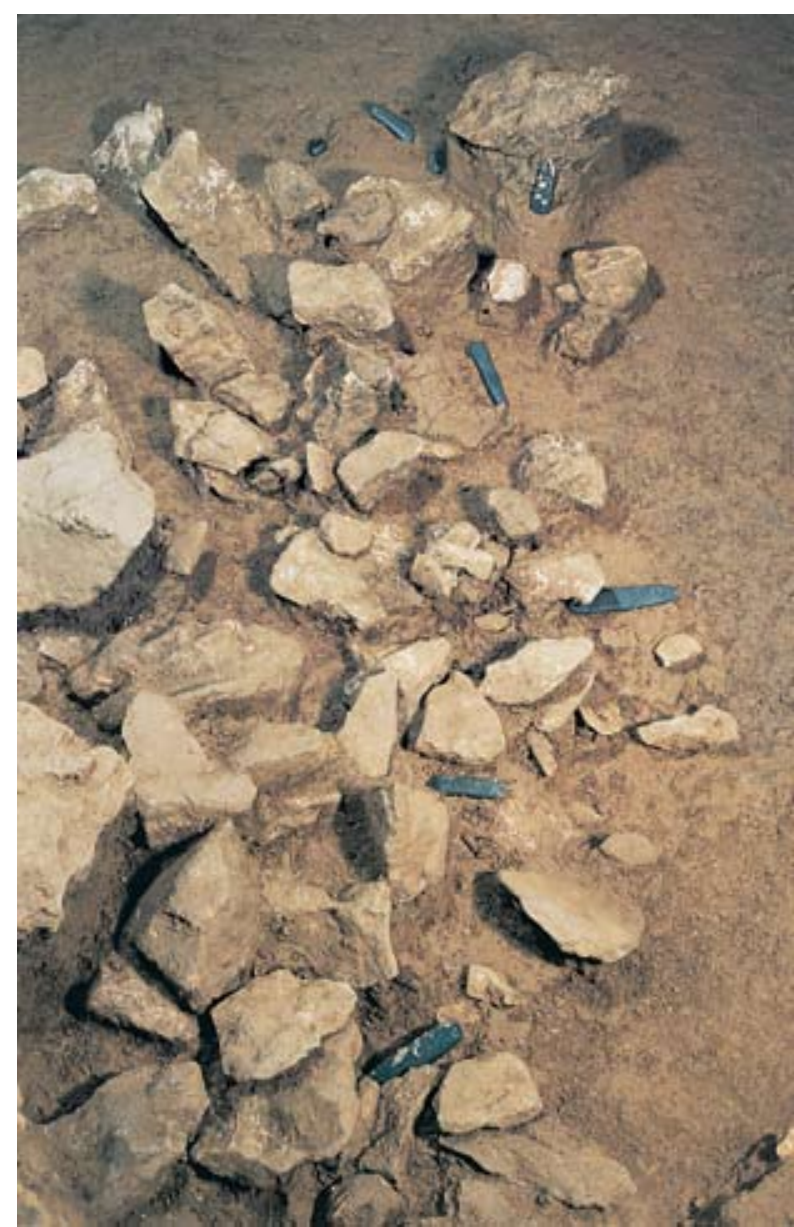

Fig. 12. Agrupación de colgantes líticos en la primera sala interior (X. Otero). / Group of lithic pendants in the first inner room $(X$. Otero).

La casi inexistencia de industrias en esta primera sala, aún más escasas que en el vestíbulo, unido a la abundancia de elementos singulares, peculiares como los colgantes, hace que nos planteemos algunas cuestiones sobre la utilización de estos dos espacios contiguos: el vestíbulo y la primera sala interior. A pesar de que las condiciones de habitabilidad del primero de ellos son aparentemente mejores, la escasez de restos apunta a una reducida actividad, además de breve -quizás esporádica-, muy probablemente centrada en el área más próxima al hogar. En cuanto a la primera sala, con mínimos restos, salvo los numerosos colgantes (Figs. 12 a 17), hace pensar en la utilización de este espacio, y por extensión quizás del resto de la cueva, como lugar de posibles actividades de tipo cultual o ritual en el Magdalenense Inferior, aunque también tuvieran lugar otras de tipo cotidiano relacionadas con la supervivencia, principalmente en la zona más luminosa del vestíbulo.

Tras la efímera ocupación del Magdaleniense Inferior la cavidad fue visitada en las fases posteriores del Magdaleniense, aunque únicamente la correspondiente a la 

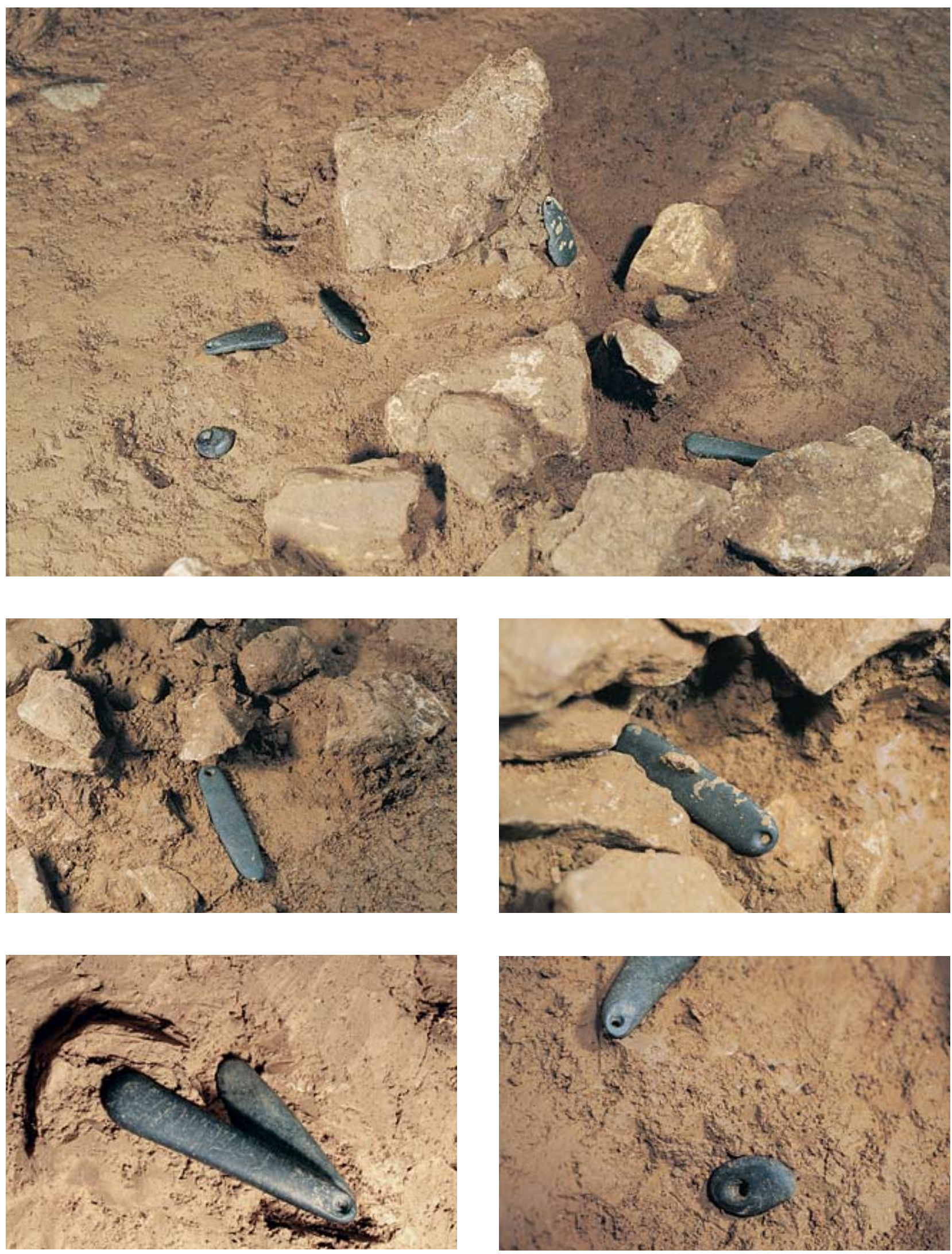

Fig. 13-17. Diversos aspectos del hallazgo de colgantes líticos en la primera sala interior (13, 14 y 15: X. Otero). / Different views of the discovery of the lithic pendants in the first inner room (13, 14 and 15: X. Otero). 


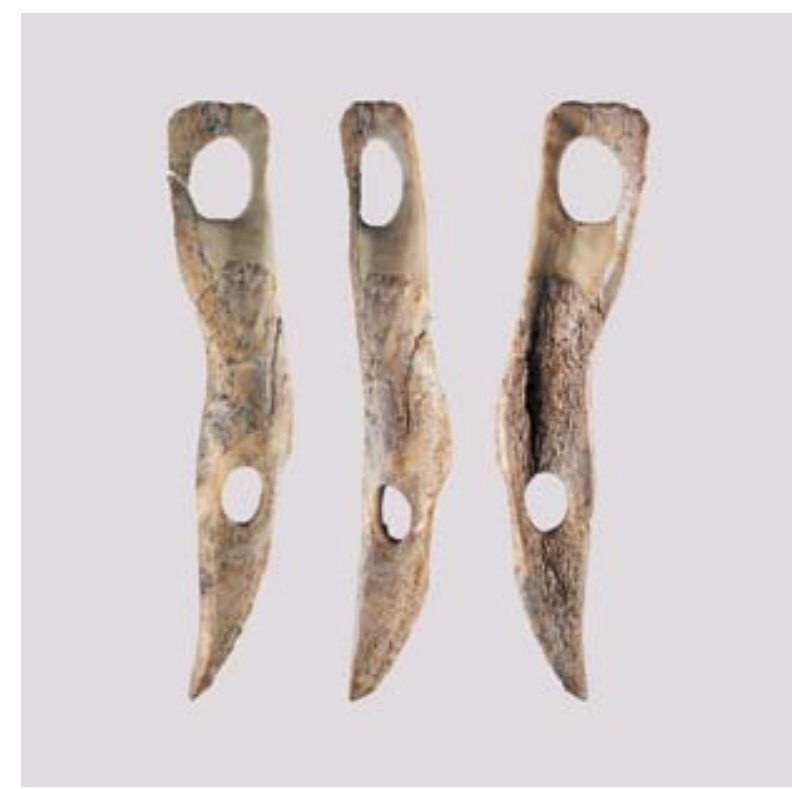

Fig. 18. Bastón perforado magdaleniense hallado en el vestíbulo de la cueva (X. Otero). / Magdalenian perforated baton found at the vestibule of the cave (X. Otero).

fase Superior-Final aporta vestigios característicos. Se identifica en la zona de tránsito y en el vestíbulo donde se presenta en un nivel de escasa potencia estratigráfica, que ha aportado al menos la fecha de 12920 \pm 50 BP, y que se ha documentado a través de un reducido número de objetos líticos y óseos, entre los que destacan un arpón de una hilera de dientes y un singular bastón perforado (Fig. 18).

Durante el Epipaleolítico el ser humano se movió por todas las zonas de la cueva, desde el vestíbulo hasta la segunda sala interior, y también existe alguna otra po-

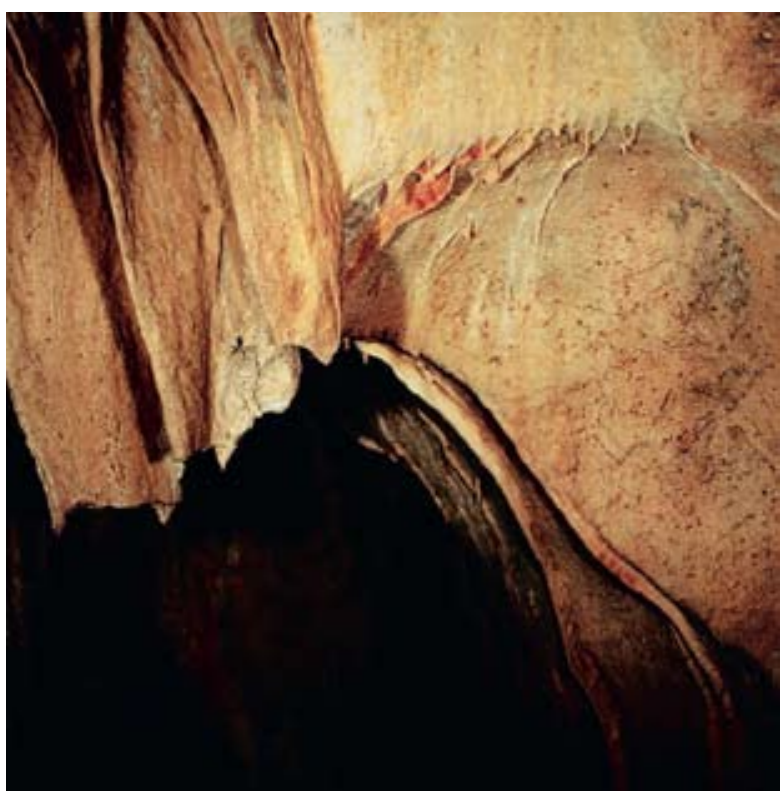

sible evidencia en el rellano exterior existente a un lado de la boca. Estas ocupaciones, si bien apenas dejaron restos industriales, nos legaron dos hogares en buen estado de conservación. Uno de ellos es una cubeta de dimensiones considerables con abundantes carbones y una capa de entre 2 y $3 \mathrm{~cm}$ de arcilla enrojecida por el fuego que llega a tener unos 2 metros de diámetro y que se ubica en el tránsito entre el vestíbulo y la primera sala interior. Sorprende, dada su envergadura, el emplazamiento seleccionado para prender esta hoguera, un pasillo de entre 2,80 m de ancho en su zona central y 4,50 m en su desembocadura a la primera sala, y una altura de tan solo 1,10 m; y además, que a su alrededor, en el contexto más próximo, no conservara restos líticos ni óseos (afectados por el fuego o no). Las muestras de carbón vegetal han sido datadas en el 8940ะ50 BP

Por otra parte, bajo este hogar y a su alrededor (bandas C', D', E' y F'), pero ya dentro de la primera sala, se recogieron dentro de un sedimento de características distintas a las del hogar, en tierra de color marrón, pequeños caracoles marinos no perforados, concretamente 193 Littorina obtusata y 42 Littorina saxatilis, y que fueron datadas en un momento anterior del Epipaleolítico -Aziliense-.

El segundo de los hogares, excavado en cubeta dentro de la arcilla amarilla que contenía los colgantes líticos del Magdaleniense Inferior, se ubica muy centrado dentro de la mitad norte de la primera sala. Su forma es circular, siendo su diámetro de 0,80 m y la profundidad máxima de $15-20 \mathrm{~cm}$ en su zona central. En su lado occidental presentaba 2 bloques colocados contiguos, de 0,70 m de longitud, y que pudieran haber servido como asientos. Dos muestras de carbón enviadas a datar han aportado Ios siguientes resultados: $8840 \pm 45$ y $8845 \pm 40 \mathrm{BP}$

Por otra parte, en la segunda sala interior, en un espacio totalmente oscuro, se han localizado abundantes

Fig. 19-20. Pinturas rupestres de la cueva de Praileaitz I (X. Otero). / Cave paintings from Praileaitz I cave (X. Otero).

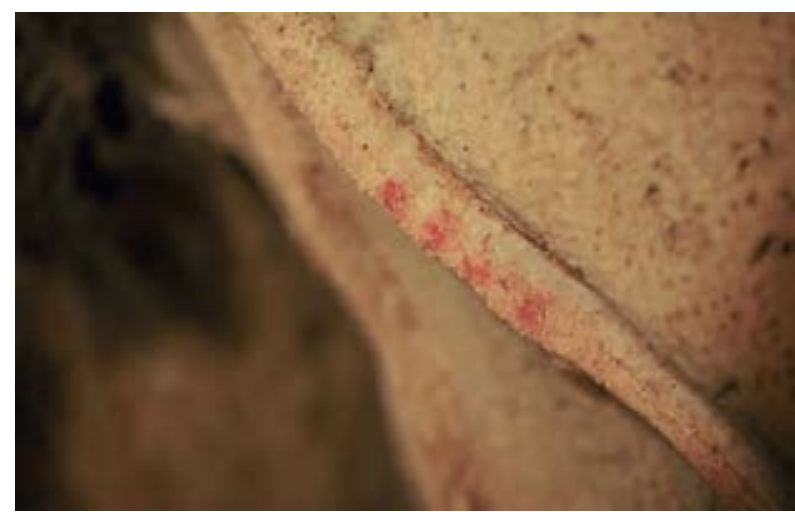


fragmentos de carbón asociados a pequeños trozos de tierra roja, distribuidos irregularmente -sin llegar a formar una mancha homogénea- en un espesor de 0,30-0,50 $\mathrm{m}$. Estos fueron datados por C14 entre el $8800 \pm 35$ y el $9225 \pm 40 \mathrm{BP}$, por lo que parecen estar en estrecha relación con los hogares descritos. Teniendo en cuenta la ausencia de continuidad del sedimento arcilloso -rojizo por su contacto directo con el fuego-, las características de la distribución y/o frecuencia de los carbones y su emplazamiento a una cota inferior a los otros hogares, nos aventuramos a proponer que hubieran podido ser transportados, por ejemplo por el agua, en periodos de elevada pluviosidad hasta esa segunda sala, o arrastrados por el paso de animales, o simplemente arrojados por el ser humano para vaciar la cubeta en alguno de los episodios en los que se prendió fuego.

Por último, aunque sin poder dar una cronología precisa, a unos $40 \mathrm{~m}$ al sur de la entrada se localizan diversos grupos de pinturas en rojo, consistentes en líneas de puntos dispuestas sobre distintos soportes (Figs. 19 y 20), que evidencian la ocupación de las zonas más profundas de la cavidad, en algún momento del Paleolítico Superior.

\section{AGRADECIMIENTOS}

J.A. Mujika-Alustiza forma parte del Grupo de Investigación del Sistema Universitario Vasco IT-622-13.

\section{BIBLIOGRAFÍA}

AGUIRRE, M.

2000 El yacimiento paleolítico de Antoliñako Koba (GautegizArteaga, Bizkaia): secuencia estratigráfica y dinámica industrial. Avance de las campañas de excavación 1995 2000. Illunzar 98/00 4, 39-81.

AGUIRRE, M., LÓPEZ QUINTANA, J.C., SÁENZ DE BURUAGA, A.

2000 Medio ambiente, industrias y poblamiento prehistórico en Urdaibai. Illunzar 98/00 4, 13-38.

ALTUNA, J., MERINO, J. M.

1984 El yacimiento prehistórico de la cueva de Ekain (Deba, Guipúzcoa). Eusko lkaskuntza. Donostia.

ALTUNA, J., BALDEÓN, A., MARIEZKUURRENA, K.

1985 Estructuras halladas en el yacimiento de Erralla. Munibe Antropologia-Arkeologia 37, 187-194.

ARAMBOUROU, R. et al.

1978 Le gisement préhistorique de Dunthy à Sorde-L'Abbaye (Landes). Mémoires de la Société Préhistorique Français 13.

ARIAS, P., ONTAÑON, R., ÁlVAREZ, E., APARICIO, M.T., CHAUVIN, A., CLEMENTE, I., CUETO, M., GONZÁLEZ URQUIJO, J.E., IBÁÑEZ, J.J., TAPIA, J., TEIRA, L.

2005 La estructura Magdaleniense de La Garma A. Aproxima- ción a la organización espacial de un hábitat paleolítico, en $\mathrm{BICHO}, \mathrm{N}$. (ed.). O Paleolítico. Actas do IV Congreso de Arqueología Peninsular (Faro 14 a 19 de septiembre de 2004). 123-143. (Promontoria Monográfica; 02).

CORCHÓN, S.

1995 Reflexiones acerca de la cronología del Magdaleniense Cantábrico. Zephyrus XLVIII, 3-19.

CHAPA, T.

1975 Magdaleniense medio y superior de Cueto de la Mina. Boletín del Real Instituto de Estudios Asturianos 86, 755-780.

DACHARY, M.

200625 années de recherches sur le Magdalénien dans les Pyrénées Occidentales, en CHAUCHAT, Cl. Préhistoire du Bassin de I'Adour. 22-27. Izpegi. Baïgorry.

DACHARY, M., CHAUVIERE, FR.-X., COSTAMAGNO, S., DAULNY, L., EASTHAM, A., FERRIER, C., FRITZ, C.

2008 La grotte Bourrouilla à Arancou (Pyrénées-Atlantiques): une sequence clef de la fin du Magdalénien pyrénéocantabrique, en JAUBERT, J., BORDES, J.G. (eds.). Les sociétés du Paléolithique dans un Grand Sud-Ouest de la France: nouveaux gisements, nouveaux résultats, nouvelles méthodes. 355-370. Société Préhistorique Française.

LEROI-GOURHAN, A.

1984 Simbolos, Artes y Creencias de la Prehistoria. Istmo. Madrid.

MERINO, J. M M $^{2}$

1984 Industria lítica del yacimiento Ekain, en ALTUNA, J., MERINO, J.M. El yacimiento prehistórico de la Cueva de Ekain (Deba, Guipúzkoa). 65-175. Sociedad de Estudios Vascos, Sociedad de Ciencias Aranzadi. Donostia.

ONTAÑÓN, R.

2003 Sols et structures d'habitat du Paléolithique supérieur. nouvelles donnés depuis les Cantabres : la Galerie Inférieure de La Garma (Cantabre, Espagne). L'Anthropologie 107(3), 333-363.

PEÑALVER, $X$.

2013-2014a La cueva de Praileaitz I (Deba, Gipuzkoa). Arkeoikuska 12, 13: 275-277, 293-294.

2014b Praileaitz I haitzuloa. La cueva de Praileaitz I (Deba, Gipuzkoa). Gipuzkoako Foru Aldundia. Donostia. (Arkeologia; 4).

PEÑALVER, X., MUJIKA, J.A.

2003 Suelo de ocupación magdaleniense en la cueva de Praile Aitz I (Deba, Gipuzkoa): evidencias de arte mobiliar. Veleia 20, 157-181.

2005a La cuestión de las azagayas de base hendida magdalenienses en la Cornisa Cantábrica. Veleia 22, 9-20.

2005b Praile Aitz I (Deba, Gipuzkoa): evidencias arqueológicas y organización espacial en un suelo Magdaleniense, en $\mathrm{BICHO}, \mathrm{N}$. (ed.) O Paleolítico. Actas do IV Congreso de Arqueología Peninsular (Faro 14 a 19 de septiembre de 2004). 143-157. (Promontoria Monográfica 02). 
PEÑALVER, X., SAN JOSE, S., MUJIKA-ALUSTIZA, J.A.

2006 Praileaitz I haitzuloko (Deba) Madeleine aldiko zintzilikarioak. Los colgantes Magdalenienses de la cueva de Praileaitz I. Gipuzkoako Foru Aldundia. Donostia. (Bertan; 22).

2017 El yacimiento arqueológico de Praileaitz I (Deba, Gipuzkoa). Metodología de la excavación, estratigrafía estructuras y dataciones arqueológicas, en PEÑALVER, X., SAN JOSE, S., MUJIKA-ALUSTIZA, J.A. La cueva de Praileaitz I (Deba, Gipuzkoa, Euskal Herria). Intervención arqueológica 2000 - 2009. Munibe Monographs. Anthropology and Archaeology Series 1, 33-120.

SAINT-PÉRIER, R.

1930 La grotte d'Isturitz I. Le Magdalénien de la Salle de SaintMartin. Masson. Paris. (Archives de l'Institut de Paléontologie Humaine ; 7).
STRAUS, L. G.

1998 Les derniers chasseurs de Rennes du monde Pyrénéen: l'abri Dufaure: un gisement tardiglaciaire en Gascogne (Fouilles 1980-1984). Bulletin de la Société Préhistorique Française 95 1, 108-109.

UTRILLA, P.

1990 La llamada «facies del País Vasco» del magdaleniense inferior cantábrico. Apuntes estadísticos. Munibe (Antropologia-Arkeologia 42, 41-54.

VEGA DEL SELLA, C.

1930 Las cuevas de la Riera y Balmori (Asturias). Memoria 38, Comisión de Investigaciones Paleontológicas y Prehistóricas. Madrid. 
